Meta

Journal des traducteurs

Translators' Journal

\title{
Listes terminologiques relatives au vocabulaire de la télédétection aérospatiale
}

\section{Serge Paul}

Volume 33, numéro 3, septembre 1988

URI : https://id.erudit.org/iderudit/003800ar

DOI : https://doi.org/10.7202/003800ar

Aller au sommaire du numéro

Éditeur(s)

Les Presses de l'Université de Montréal

ISSN

0026-0452 (imprimé)

1492-1421 (numérique)

Découvrir la revue

Citer cet article

Paul, S. (1988). Listes terminologiques relatives au vocabulaire de la télédétection aérospatiale. Meta, 33(3), 416-427.

https://doi.org/10.7202/003800ar d'utilisation que vous pouvez consulter en ligne. 


\section{LISTES TERMINOLOGIQUES RELATIVES AU VOCABULAIRE DE LA TÉLÉDÉTECTION AÉROSPATIALE}

ARRÊTÉ DU 31 MARS 1987 - JOURNAL OFFICIEL DE LA RÉPUBLIQUE FRANÇAISE

Le $5^{\mathrm{e}}$ arrêté de terminologie consacré essentiellement à l'enrichissement du vocabulaire RADAR dont un type de matériel, le radar à antennes latérales, présente une imagerie du plus haut intérêt pour la reconnaissance de cibles terrestres ou planétaires.

Ce vocabulaire présentait de nombreuses lacunes en langue française bien que l'expérimentation du radar aéroporté, en France, remonte au début des années 1970 et que celle d'un radar spatioporté, américain (SEASAT, SIR-A et B) a eu lieu ces der- 
nières années. De plus, la technique du radar à synthèse d'ouverture est en plein développement ; dans moins de deux ans, le projet E.R.S.-1 de l'Agence spatiale européenne, au sein de laquelle la France a une participation importante, arrivera à terme.

Dans cet arrêté de terminologie, les deux tiers des mots traités, une quarantaine d'articles (Annexe I), relèvent directement de ce domaine spécifique et, de ce fait, il est pourvu au remplacement des termes et sigles anglo-américains correspondants (Annexe III). La liste des besoins n'est cependant pas épuisée ; une vingtaine d'articles sont en cours de traitement.

Notre effort a également porté sur les sigles qui foisonnent en anglo-américain ; par ricochet, le jargon technique en est affecté. Notre politique répond à celle de l'Académie française sur ce sujet car l'abus de la siglaison oblitère la diffusion des connaissances à un public non initié. Seuls, dix sigles français sont officialisés, avec leurs équivalents en langue anglaise (Annexe II).

\section{ANNEXE I}

avers de pente radar, $n . m$.

Domaine : Télédétection électromagnétique.

Définition : Partie du terrain faisant face au front d'onde.

Voir aussi : ombre de radar, revers de pente radar.

Anglo-américain : radar foreslope.

bathymètre à lidar, n.m.

Domaine : Télédétection électromagnétique.

Définition : Instrument destiné à la mesure de profondeurs sous-marines à l'aide d'un laser.

Anglo-américain : lidar bathymeter.

détection et télémétrie par la lumière, n.f.*

Voir : lidar.

diagramme d'antenne, n.m.

Voir : diagramme de gain d'antenne.

diagramme de gain d'antenne, n.m.

Abréviation : diagramme d'antenne, n.m.

Domaine : Télédétection électromagnétique/Technologie des hyperfréquences.

Définition : Représentation graphique plane du gain directionnel d'une antenne.

Note : 1. Généralement, cette représentation est faite suivant les plans de sections principales.

2. Conventionnellement, on peut rapporter les amplitudes de ce diagramme de gain, soit au gain de l'antenne isotrope équivalente, soit à la valeur maximale du gain obtenu ; dans ce dernier cas, on parle de diagramme relatif.

Voir aussi : lobe d'antenne.

Anglo-américain : antenna pattern.

\section{distorsion d'écho, n.f.}

Domaine : Télédétection électromagnétique.

Définition; Altération du signal reçu, résultant de phénomènes qui influencent la propagation des ondes électromagnétiques.

Anglo-américain : echo distortion. 


\section{donnée en polarisations croisées, $n . f$.}

Domaine : Télédétection électromagnétique.

Définition : Donnée obtenue dans un plan de polarisation orthogonal à celui du signal émis.

Voir aussi : donnée en polarisations parallèles, image-radar.

Anglo-américain : cross polarization data.

\section{donnée en polarisations parallèles, n.f.}

Domaine : Télédétection électromagnétique.

Définition : Donnée obtenue dans un plan de polarisation parallèle à celui du signal émis.

Voir aussi : donnée en polarisations croisées, image-radar.

Anglo-américain : like polarization data.

\section{écho-fantôme, n.m.}

Domaine : Télédétection électromagnétique.

Définition : Écho supplémentaire provoqué par une réflexion parasite sur une cible, qui fait percevoir l'image virtuelle d'une autre cible.

Anglo-américain : ghost echo.

\section{écho-mirage, n.m.}

Domaine : Télédétection électromagnétique.

Définition : Écho d'objets non visibles ou non identifiés, provoqué par certaines conditions atmosphériques.

Voir aussi : trajet multiple.

Anglo-américain : angel echo.

\section{fluorescence excitée par laser, n.f.}

Voir : fluorodétecteur à laser.

\section{fluorodétecteur à laser, n.m.}

Domaine : Télédétection électromagnétique.

Définition : Capteur actif destiné à détecter et, au besoin, à localiser certaines cibles d'après leur fluorescence excitée par un faisceau laser.

Note : L'expression anglo-américaine laser induced fluorescence (abrév. L.I.F.), qui se traduit par fluorescence excitée par laser, désigne le phénomène physique détecté.

Anglo-américain : laser fluorosensor.

\section{fluorodétecteur en raies de Fraunhofer, n.m.}

Domaine : Télédétection électromagnétique.

Définition : Capteur passif destiné à détecter dans les raies de Fraunhofer la fluorescence de certaines cibles, excitée par le rayonnement solaire.

Anglo-américain : Fraunhofer line discriminator (F.L.D.).

\section{image basse fréquence, $n . f$.}

Voir : image de basse fréquence.

\section{image chronoséquentielle, n.f.}

Domaine : Télédétection électromagnétique.

Définition : Image résultant d'un taitement de données télédétectées saisies sur la même scène à plusieurs moments du cycle nychthéméral avec le(s) mème(s) capteur(s).

Voir aussi : image multidate. 
image de basse fréquence, n.f.

Abréviation : image basse fréquence, n.f.

Domaine : Télédétection.

Définition : Image qui ne contient plus que les harmoniques inférieures d'une transformée de Fourier bidimensionnelle.

Voir aussi : image de haute fréquence.

Anglo-américain : low frequency image.

image de haute fréquence, n.f.

Abréviation : image haute fréquence, n.f.

Domaine : Télédétection.

Définition : Image qui ne contient plus que les harmoniques supérieures d'une transformée de Fourier bidimensionnelle.

Voir aussi : image de basse fréquence.

Anglo-américain : high frequency image.

image diachronique, n.f.

Voir : image multidate.

image filtrée passe-bas, n.f.

Abréviation : image passe-bas, n.f.

Domaine : Télédétection.

Définition : Image filtrée dont on ne conserve que les termes de basse fréquence d'une transformée de Fourier bidimensionnelle.

Voir aussi : image filtrée passe-haut.

Anglo-américain : low-pass image.

image filtrée passe-haut, n.f.

Abréviation : image passe-haut, n.f.

Domaine : Télédétection.

Définition : Image filtrée dont on ne conserve que les termes de haute fréquence d'une transformée de Fourier bidimensionnelle.

Voir aussi : image filtrée passe-bas.

Anglo-américain : high-pass image.

image haute fréquence, $n . f$.

Voir : image de haute fréquence.

image multidate, n.f.

Domaine : Télédétection électromagnétique.

Synonyme : image diachronique, n.f.

Définition : Image résultant d'un traitement de données télédétectées saisies sur une même scène à des jours ou des époques différents.

Note : 1. Les saisies ne sont pas obligatoirement effectuées avec le(s) même(s) capteur(s).

2. Le terme "image multitemporelle " doit être évité en raison de la non-pluralité du temps.

Voir aussi : image chronoséquentielle.

Anglo-américain : multidate image, multitemporal image.

image passe-bas, n.f.

Voir : image filtrée passe-bas. 


\section{image passe-haut, n.f.}

Voir : image filtrée passe-haut.

\section{image plurielle par radar, n.f.}

Abréviation : image radar plurielle, n.f.

Domaine : Télédétection électromagnétique.

Définition : *Image-radar d'une cible résultant du traitement simultané des signaux en provenance de plusieurs impulsions.

Anglo-américain : radar multiple-look image, radar multi-look image.

\section{image-radar, n.f.}

Domaine : Télédétection électromagnétique.

Définition : Document obtenu par ${ }^{*}$ radargraphie.

Notes : 1. En choisissant les polarisations à l'émission et à la réception, on peut obtenir plusieurs imagesradar différentes, par exemple en polarisation rectiligne, quatre images que l'on spécifie généralement par les abréviations HH ou VH dans le cas de polarisations parallèles, horizontales ou verticales respectivement, et $\mathrm{HV}$ ou VH dans le cas de polarisations croisées.

2. La première lettre de l'abréviation rappelle la polarisation à l'émission, la seconde lettre la polarisation à la réception.

Voir aussi : image plurielle de radar, image simple de radar, donnée en polarisations croisées, donnée en polarisations parallèles.

Anglo-américain : radar image.

\section{image radar plurielle, n.f.}

Voir : image plurielle par radar.

\section{image radar simple, n.f.}

Voir : image simple par radar.

\section{image simple par radar, n.f.}

Abréviation : image radar simple, n.f.

Domaine : Télédétection électromagnétique.

Définition : *Image-radar d'une cible obtenue à partir d'une seule impulsion.

Note : Dans le cas du R.S.O., une telle image peut être obtenue par la composition de signaux cohérents reçus par l'antenne synthétique.

Anglo-américain : radar single-look image.

\section{imagette, n.f.}

Domaine : Télédétection électromagnétique.

Définition : Partie d'image identifiée par un point d'appui ou un élément de repérage.

Voir aussi : subimage.

\section{lidar, n.m.}

Domaine : Télédétection électromagnétique.

Définition : Capteur actif utilisant comme source émettrice un laser.

Notes : 1. Le terme lidar est l'acronyme de l'expression anglo-américaine light detection and ranging, qui se traduit en français par détection et télémétrie par la lumière.

2. Le lidar est utilisé pour la mesure des distances, la détection et, éventuellement, la localisation de constituants des milieux rencontrés.

Anglo-américain : lidar. 


\section{lidar atmosphérique, n.m.}

Domaine : Télédétection électromagnétique.

Définition : *Lidar destiné à la détection et, au besoin, à la localisation de polluants et de certains constituants de l'environnement atmosphérique.

Anglo-américain : atmospheric lidar.

\section{lidar imageur, n.m.}

Domaine : Télédétection électromagnétique.

Définition : Capteur actif imageur utilisant comme source émettrice un laser.

Note : Le terme "radar à laser " parfois employé comme équivalent de l'expression anglo-américaine laser radar est à proscrire en raison du caractère contradictoire de ces deux notions.

Anglo-américain : laser radar.

\section{lobe d'antenne, n.m.}

Domaine : Télédétection électromagnétique/Technologie des hyperfréquences.

Définition : Volume spécifique à chaque antenne, qui représente les caractéristiques directionnelles d'émission (ou de réception) de celle-ci.

Notes : 1. Dans l'hypothèse d'une antenne isotrope, ce volume serait une sphère centrée sur le dipôle ou le cornet émetteur.

2. Dans le cas d'une antenne anisotrope, par suite des phénomènes de diffraction, ce volume se décompose en lobes principaux (a.a. main/major lobes) et en lobes secondaires ou lobes latéraux (a.a. secundary/minor ou side lobes).

Dans les lobes principaux transite la part maximale du rayonnement émis (ou reçu). On appelle axe d'un lobe principal la direction dans laquelle le gain d'antenne est maximal ; cette direction constitue l'axe de visée.

Dans les lobes secondaires transite la part restante du rayonnement émis (ou reçu). Les lobes secondaires sont des sources d'émission (ou de réception) dont on cherche à réduire les effets perturbateurs.

Anglo-américain : antenna lobe.

\section{lobe latéral, n.m.}

Voir : lobe d'antenne.

lobe principal, n.m.

Voir : lobe d'antenne.

lobe secondaire, n.m.

Voir : lobe d'antenne.

\section{ombre de radar, n.f.}

Abréviation : ombre-radar, n.f.

Domaine : Télédétection électromagnétique.

Définition : Étendue de terrain non éclairée par l'onde radar émise, par suite de l'interception de tout ou partie du faisceau par un obstacle et de l'absence de diffusion atmosphérique.

Note : En présence d'un obstacle déterminé, la forme et la dimension de l'ombre-radar dépendent en particulier de l'angle de dépression du capteur et de la direction de vol et de la plate-forme.

Voir aussi : effet de rapprochement, déversement en radar, revers de pente radar.

Anglo-américain : radar shadow. 
ombre-radar, n.f.

Voir : ombre de radar.

prétraitement d'image, n.m.

Domaine : Télédétection.

Domaine : Opération(s) conduite(s) sur les données brutes destinée(s) à rendre celles-ci aptes à une analyse thématique qui exige, le plus souvent, un traitement.

Note : Ces opérations consistent, essentiellement, à effectuer des formatages, le délignage, des corrections géométriques, radiométriques, des rééchantillonnages.

Voir aussi : donnée corrigée, donnée élaborée, donnée prétraitée, niveau de prétraitement, rectification d'image.

Anglo-américain : image preprocessing.

\section{radargrammétrie, n.f.}

Domaine : ,Télédétection électromagnétique.

Définition : Techniques d'exploitation des *images-radar qui permettent de déterminer les caractéristiques géométriques du terrain et d'en représenter des formes ainsi que des détails.

Voir aussi : stéréoradargrammétrie.

Anglo-américain : radargrammetry.

radargraphie, n.f.

Domaine : Télédétection électromagnétique.

Définitions : 1. Technique qui permet d'obtenir des images à partir des signaux recueillis par un radar imageur.

2. Document obtenu par cette technique.

Voir aussi : image-radar.

Anglo-américain : radar image.

\section{radiométrie spectrale imageante, n.f.}

Abréviation : R.S.I., n.f.

Domaine : Télédétection électromagnétique.

Synonyme : spectroradiométrie imageante, n.f.

Définition : Utilisation de la radiométrie spectrale pour obtenir des images potentielles ou réelles d'une scène.

Anglo-américain : imaging spectrometry.

\section{réponse spectrale, n.f.}

Domaine : Télédétection électromagnétique.

Définition : Résultat de l'interaction, en fonction de la longueur d'onde, d'un rayonnement incident donné et de la cible irradiée qui intervient par ses caractéristiques physiques.

Anglo-américain : spectral response.

\section{revers de pente radar, n.m.}

Domaine : Télédétection électromagnétique.

Définition : Partie du terrain au revers de celle qui fait face au front d'onde.

Voir aussi : ombre de radar, avers de pente radar.

Anglo-américain : radar backslope.

\section{R.S.I., n.f.}

Voir : radiométrie spectrale imageante. 


\section{section efficace du diagramme de gain, n.f.}

Domaine : Télédétection électromagnétique/Technologie des hyperfréquences.

Synonyme : section efficace en radar, n.f.

Définition : Produit de l'aire apparente de la cible dans la direction du faisceau par le gain effectif de la cible considérée comme un radiateur dans cette direction.

Anglo-américain : radar cross section.

section efficace en radar, n.f.

Voir : section efficace du diagramme de gain.

signal second, n.m.

Domaine : Télédétection électromagnétique.

Définition : Écho supplémentaire reçu après un délai supérieur à la période de récurrence des impulsions du radar.

Voir aussi : trajet multiple.

Anglo-américain : second go around.

sous-scène, n.f.

Domaine : Télédétection électromagnétique.

Définition : Sous-ensemble d'une scène issu d'une partie du groupe-capteur.

Anglo-américain : sub scene.

\section{spatiocarte, n.f.}

Domaine : Télédétection électromagnétique.

Définition : Carte obtenue à partir de données recueillies par les satellites d'observation de la Terre, combinées éventuellement avec des éléments tirés de cartes au trait existantes.

Note : Ce terme peut s'appliquer à des cartes d'autres planètes et satellites naturels, obtenues dans les mêmes conditions.

Anglo-américain : satellite image map, space map.

spectroradiométrie imageante, n.f.

Voir : radiométrie spectrale imageante.

\section{stéréoradargrammétrie, n.f.}

Domaine : Télédétection électromagnétique.

Définition : Techniques d'exploitation d'un couple d'images-radar impliquant la corrélation visuelle ou instrumentale des points homologues de ce couple.

Note : On distingue la stéréoradarscopie dans le cas d'une exploitation visuelle et la stéréoradargraphie dans celui d'une exploitation graphique.

Voir aussi : radargrammétrie.

Anglo-américain : stereoradargrammetry.

stéréoradargraphie, n.f.

Voir : stéréoradargrammétrie.

stéréoradarscopie, n.f.

Voir : stéréoradargrammétrie. 


\section{stéréoradiométrie, n.f.}

Domaine : Télédétection électromagnétique.

Définition : Technique qui combine le traitement de données radiométriques provenant d'un enregistrement stéréoscopique de la scène avec la corrélation visuelle ou instrumentale des points homologues de ce couple stéréoscopique.

Voir aussi : stéréoradargrammétrie.

Anglo-américain : stereoradiometry.

\section{subimage, n.f.}

Domaine : Télédétection électromagnétique.

Définition : Subdivision d'une image ou sous-ensemble dans cette image.

Voir aussi : imagette.

Anglo-américain : sub image.

\section{trajet multiple, n.m.}

Domaine : Télédétection/Technologie des hyperfréquences.

Définition : Ensemble des divers chemins empruntés par un même signal entre l'émetteur et le récepteur. Note : La multiplicité de ces chemins résulte principalement de l'hétérogénéité de l'atmosphère et des conditions de réflexion sur les obstacles, provoquant une multiplication des échos.

Voir aussi : écho-mirage, signal second.

Anglo-américain : multiple path.

\section{ANNEXE II}

\section{F.R.I.}

Domaine : T.D.E.M./Technologie des hyperfréquences. Explicitation : Fréquence de Récurrence des Impulsions. Anglo-américain : Pulse Repetition Frequency (P.R.F.).

\section{H.R.V.}

Domaine : Télédétection électromagnétique.

Explicitation : à Haute Résolution dans le Visible

Anglo-américain : High Resolution in the Visible (H.R.V.).

\section{I.R.C.}

Domaine : Télédétection électromagnétique.

Explicitation : InfraRouge Couleur.

Anglo-américain : Colo(u)r Infra-Red (C.I.R.).

\section{R.A.A.S.}

Domaine : Télédétection électromagnétique.

Explicitation : Radar à Antenne Synthétique.

Synonyme R.S.O. : Radar à Synthèse d'Ouverture.

Anglo-américain : Synthetic Aperture Radar (S.A.R.).

\section{R.S.O.}

Voir : R.A.A.S. 
R.V.L.

Domaine : Télédétection électromagnétique.

Explicitation : Radar à Visée Latérale.

Anglo-américain : Side-looking Radar (S.L.R.).

S.M.B.

Domaine : Télédétection électromagnétique.

Explicitation : Scanneur MultiBande.

Anglo-américain : MultiSpectral Scanner (M.S.S.).

\section{S.M.B.T.M.}

Domaine : Télédétection électromagnétique.

Explicitation : Scanneur MultiBande pour Thèmes Multiples.

Abréviation : T.M.

Anglo-américain : Thematic Mapper (T.M.).

\section{T.D.E.M.}

Domaine : Télédétection électromagnétique.

Explicitation : TéléDétection ÉlectroMagnétique.

Anglo-américain : Electro Magnetic Remote Sensing (E.M.R.S.)

\section{T.M.}

Voir : S.M.B.T.M.

La commission ministérielle de terminologie de la télédétection aérospatiale a également examiné une liste complémentaire de sigles d'usage courant en angloaméricain, dont la parution est prévue dans le Bulletin de la Société française de photogrammétrie et de télédétection.

ANNEXE III

\section{Index permuté anglo-américain/français}

TERMES ET SIGLES
anglo-américains
à remplacer
angel echo
antenna lobe
antenna pattern
antenna pattern
atmospheric lidar
C.I.R.
cross polarization data
echo distortion
E.M.R.S.
F.L.D.
Fraunhofer line discriminator (F.L.D.)
ghost echo
high frequency image

\section{ÉQUIVALENTS FRANÇAIS}

\author{
écho-mirage \\ lobe d'antenne \\ diagramme de gain d'antenne \\ diagramme d'antenne (abrév.) \\ lidar atmosphérique \\ I.R.C. \\ données en polarisations croisées \\ distorsion d'écho \\ T.D.E.M. \\ fluorodétecteur en raies de Fraunhofer \\ fluorodétecteur en raies de Fraunhofer \\ écho-fantôme \\ image de haute fréquence
}


high frequency image

high-pass image

high-pass image

H.R.V.

image preprocessing

imaging spectrometry

imaging spectrometry

laser fiuorosensor

laser induced fuorescence (L.I.F.)

laser radar

lidar

lidar bathymeter

L.I.F.

light detection and ranging (lidar)

like polarization data

low frequency image

low frequency image

low-pass image

low-pass image

main lobe

major lobe

minor lobe

M.S.S.

multidate image

multidate image

multiple path

multitemporal image

multitemporal image

P.R.F.

radar backslope

radar cross section

radar cross section

radar foreslope

radar image

radar image

radargrammetry

radar multiple-look image

radar multiple-look image

radar multi-look image

radar multi-look image

radar shadow

radar shadow

radar single-look image

radar single-look image

S.A.R.

S.A.R.

satellite image map

second go around

secundary lobe

side lobe

S.L.R. image haute fréquence (abrév.)

image filtrée passe-haut

image passe-haut (abrév.)

H.R.V.

prétraitement d'image

radiométrie spectrale imageante (R.S.I.)

spectroradiométrie imageante (syn.)

fluorodétecteur à laser

fluorescence excitée par laser

lidar imageur

lidar

bathymètre à lidar

fiuorescence excitée par laser

détection et télémétrie par la lumière données en polarisations parallèles

image de basse fréquence

image basse fréquence (abrév.)

image filtrée passe-bas

image passe-bas (abrév.)

lobe principal

lobe principal

lobe secondaire ou lobe latéral

S.M.B.

image multidate

image diachronique (syn.)

trajet multiple

image multidate

image diachronique (syn.)

F.R.I.

revers de pente radar

section efficace du diagramme de gain

section efficace en radar (syn.)

avers de pente radar

radargraphie

image-radar

radargrammétrie

image plurielle par radar

image radar plurielle (abrév.)

image plurielle par radar

image radar plurielle (abrév.)

ombre de radar

ombre-radar (abrév.)

image simple par radar

image radar simple (abrév.)

R.A.A.S.

R.S.O. (syn.)

spatiocarte

signal second

lobe secondaire ou latéral

lobe secondaire ou latéral

R.V.L. 


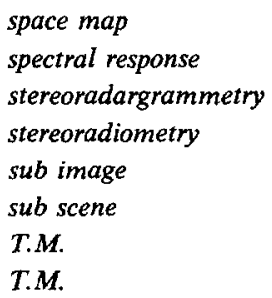

\author{
spatiocarte \\ réponse spectrale \\ stéréoradargrammétrie \\ stéréoradiométrie \\ subimage \\ sous-scène \\ S.M.B.T.M \\ T.M. (abrév.)
}

\section{ANNEXE IV}

Index des impropriétés à éviter

\section{FORMES IMPROPRES}

image multitemporelle

radar à laser

\section{FORMES RETENUES}

image multidate

lidar imageur

Professeur Serge Paul Président de la Commission de terminologie de la télédétection aérospatiale, Paris, France 